\title{
A RECONSIDERATION OF THE BROWN-DOMINANT POSITION EFFECT
}

\author{
HERMAN M. SLATIS \\ Depariment of Genetics, McGill University, Montreal, Canada \\ Received June 30, 1954
}

B

ROWN-DOMINANT $\left(b w^{D}\right)$ has been described by HiNToN $(1940,1946)$ as a mutant at the brown locus (bw, 2-104.5) in Drosophila melanogaster. It has been interpreted as being inseparable from what Schultz (see HrNToN 1942) considered to be a duplication of salivary gland chromosome band $59 \mathrm{E} 1 \cdot 2$, and although the extra material was referred to as heterochromatin by HinToN and Goodsmitu (1950), their cytological description of the mutant was substantially the same as previously reported. However, they indicate that the distal band is the normal one and the proximal band is the one which usually is heterochromatic. HinTon (1942) described $b w^{D}$ as not causing variegation, although Hinton and Goopsmith noted variegation in the heterozygote between two X-ray induced changes of the mutant. These observations resulted in the interpretation of $b w^{D}$ as a euchromatic position effect. The failure of SLATIS (1955) to produce any strong euchromatic position effects at the $b w$ locus among many due to heterochromatin prompted this investigation of $b w^{D}$.

\section{RESULTS}

\section{Cytological observations}

The cytological examination of heterozygotes between $b w^{D}$ and wild type confirmed Hinton's (1946) description of what appeared to be pairing of the normal band with either of the two bands in the duplication, although the distal band was usually paired and the proximal band only infrequently so. The chromocenter, various euchromatic regions, and chromosome tips, particularly the tip of $2 \mathrm{R}$ (which is very close to the brown locus), were attracted to $59 \mathrm{E}$.

Study of the homozygote indicates that this is an erroneous picture. In some slides the region just proximal to band $59 \mathrm{E} 1 \cdot 2$ is distorted or broken in as many as thirty percent of all cells. It is clear in almost every cell that the extra region is much more than the equivalent of $59 \mathrm{E} 1 \cdot 2$. A number of well stretched specimens agree in showing an insertion of three heavy bands just to the left of $59 \mathrm{E} 1.2$ and a single light band has been seen on rare occasion as the rightmost band of the insertion. Stress or breakage tends to occur between the extra bands and $59 \mathrm{E} 1 \cdot 2$, and occasionally pairing with heterochromatin will separate these two regions. The inserted material frequently appears to be banded, but it may show a distinct absence of bands in otherwise excellent preparations. The appearance is very much like that of the proximal regions of the long chromosome arms (see HanNaH 1951).

\section{Variegation}

The demonstration of variegation in brown locus position effects is greatly aided by combining the position effect with scarlet $(s t)$ and using the shadow technique of 
observation (SLATIS 1955). The homozygote $b w^{D}$; st is completely white in eye color in all individuals, as is the genotype $b w^{D} / b w$; st. However, the genotype $b w^{D} /+; s t$ is always strongly variegated. At emergence from the pupa the spots can only be seen with the shadow technique, although the eye is orange rather than white. Within a few days the spots have darkened to brown on a lightly pigmented background and can be seen without special techniques. The individual spots rarely exceed a single facet in size, although about one percent of the flies have a large spot of brown color which may cover any number of facets. Spots of scarlet, which would indicate normal activity at the brown locus, have not been seen. The females are distinctly more pigmented than the males and the pigment is strongest in the front of the eye in both sexes. Hinton (1942) failed to observe any variegation even with the aid of vermilion (v). On testing, it was found that $v$; bw is light orange in color rather than white (with the $v$ allele used in this experiment, and presumably with that used by HinToN) and this obscured the variegation when combined with $b w^{D}$. With the shadow technique, $v ; b w^{D} /+$ is seen to be variegated. Even with the shadow technique it is almost impossible to observe variegation in $b w^{D} /+$ flies which have not had the brown colored pigment removed by the use of mutants such as $v$ or $s t$, or at least slightly diluted by a mutant such as purple.

\section{Crossover data}

The $b w$ locus is bracketed by plexus $(p x, 2-100.5)$ and speck $(s p, 2-107.0)$, which are excellent genes for the measurement of linkage. Crossovers were measured at $25^{\circ} \mathrm{C}$ and $29^{\circ} \mathrm{C}$ for the distances $p x-b w$ and $b w-s p$ in the normal chromosomes and in heterozygotes for $b w^{D}$. The $p x$-sp distance was measured in flies homozygous for $b w^{D}$ (the $b w$ position cannot be marked phenotypically in $b w^{D}$ homozygotes). The results are shown in table 1 . None of the differences between the two temperatures are significant, so only the $25^{\circ} \mathrm{C}$ data will be studied, as they are standard and more numerous. At $25^{\circ} \mathrm{C}$ the $p x-b w$ distance of 3.87 is close to the standard map distance of 4.0, and the $b w-s p$ distance of 2.31 is close to the standard 2.5. The overall distance of $6.18 \pm 0.34$ is almost exactly one standard error from the expected value, which is not a significant departure. In flies heterozygous for $b w^{D}$ the $p x-b w$ distance decreases strongly but the bre-sp distance decreases slightly, if at all. The overall decrease $(p x-s p)$ is significant at the .001 level. The homozygote for the inserted $b w^{D}$ region

TABLE 1

The effect of temperature and genotype on crossing over in the region of the brown locus

\begin{tabular}{c|c|c|c|c|c}
\hline Genotype & ${ }^{\circ} \mathrm{C}$ & Number & \multicolumn{3}{|c}{ Crossover distance } \\
\hline & & & $p x-b w$ & $b w-s p$ & $p x-s p \pm$ S.E. \\
\hline$+/+$ & 25 & 4982 & 3.87 & 2.31 & $6.18 \pm 0.34$ \\
$+/+$ & 29 & 1609 & 3.11 & 2.55 & $5.66 \pm 0.58$ \\
$+/ b w^{D}$ & 25 & 4625 & 2.25 & 1.99 & $4.24 \pm 0.30$ \\
$+/ b w^{D}$ & 29 & 2528 & 2.29 & 2.69 & $4.98 \pm 0.43$ \\
$b w^{D} / b w^{D}$ & 25 & 5182 & - & - & $2.10 \pm 0.20$ \\
$b w^{D} / b w^{D}$ & 29 & 2030 & - & - & $2.27 \pm 0.33$ \\
\hline
\end{tabular}


greatly reduces crossing over and the $p x-s p$ distance is only 2.10 units. This is significantly different at the .001 level from both the normal and the heterozygous arrangements.

Since $b w^{D}$ might be associated with a lowering of crossing over within the entire genome, or at least within the entire second chromosome, a test was undertaken using other loci. Flies homozygous for $b w^{D}$ showed a distance of $22.0 \pm 1.0$ units based on 1727 individuals for the 27.0 units between black $(b, 2-48.5)$ and curved $(c, 2-75.5)$. Some double crossovers were probably overlooked, which might increase the 22 units to an amount which does not differ significantly from the expected value. The $c$-sp distance is made up of 25.0 units on the standard map from $c$ to $p x$, and 2.1 units from $p x$ to $s p$ in flies homozygous for $b w^{D}$. The same 1727 flies also showed exactly $22.0 \pm 1.0$ units for this distance. A group of 1344 flies were examined for $b-p x$ and $p x-s p$ crossovers in flies homozygous for $b w^{\nu}$. The $p x-s p$ distance of 2.4 units confirmed previous findings (see table 1). The $b-p x$ distance of 35.8 units was lower than the randomness expected (53.0 units on the standard map), but double crossovers would account for a large part of this.

Despite the reduction of crossing over in the region of the $b w$ locus in heterozygotes for $b w^{D}$, there is the possibility that an occasional crossover might separate the allele at the $b w$ locus from the inserted material. The results of HrNron and GoodSMITH (1950) suggest that this allele is wild type in function, so with all flies homozygous for $s t$, females $+b w^{\nu}+/ p x b w s p$, which are white in eye color, were mated with $p x b w s p$ males. A total of 20,885 flies failed to show any eye pigment, which would have indicated that a crossover had occurred which separated a wild type bw locus allele from the insertion. The cultures were allowed to form a second generation and these were also examined, since half the $F_{1}$ females would have been of the same genotype as their mothers (although one quarter of the appropriate crossover type offspring might be difficult to observe because of combination with a $b w^{D}$ sperm). No crossovers were found among $31,250 \mathrm{~F}_{2}$ flies.

\section{DISCUSSION}

Cytologically, the study of brown-dominant is hampered by the investigation of structurally heterozygous animals. In the heterozygote the inserted material is squeezed into a small space, particularly in the center of the chromosome. As both it and band $59 \mathrm{E} 1 \cdot 2$ are heavily stained, it is frequently difficult to determine which of the two is paired with the single dark band in the normal chromosome. Either an accidental displacement or an optical illusion (but more probably the latter) occasionally gives the impression that the inserted material is paired with the normal band and that the adjacent $59 \mathrm{E} 1 \cdot 2$ band is unpaired. In the case of a small deficiency in this region (unpublished observations) a small number of cells showed a similar optical illusion in which a band whose homologue was deficient paired with the other half of an intact band.

On the other hand, the study of the homozygote clearly shows that the inserted region is not equivalent to band $59 \mathrm{E} 1 \cdot 2$, being much longer and physically distinctive. Also, the insertion produces variegation in certain genotypes. Because of these facts, 
the inserted material may reasonably be assumed to be chromocentral heterochromatin, and the $b w^{\nu}$ position effect is no different in its action than any of the many heterochromatic position effects which have already been studied at the brown locus (see Statis 1955).

Brown-dominant is the first mutant at this locus which clearly indicates that the variegated position effects are caused by the heterochromatin rather than by the centromere, which is usually associated with it. It is of particular interest in view of the recent study which McClintock (1951) has made of the data on white-mottled 5 which had been collected by GrIFFen and Stone (1940). MCClintock believes that many of the further rearrangements induced in this mutant must have included a small part of the heterochromatin of chromosome 4 . MCClintock has noticed that in certain cases these translocations were to the end of a chromosome arm and did not produce variegation, but further breakage and translocation nearer to a centromere returned the ability to produce variegation. The observation that $b w^{D}$ is related to chromocentral heterochromatin supports MCCLINTOCK's contention that heterochromatin was associated with many of the translocations despite the fact that it was not cytologically recognizable. However, the $b w^{D}$ mutant is remarkably strong since it not only suppresses the wild allele in the same chromosome, but it almost completely suppresses the wild allele in the homologue, and this strength is manifested at the end of a chromosome arm. None of the $R(+)$ alleles found by SLATIS (1955) had as great an effect on the wild type allele in the same chromosome and none of the $R(b w)$ alleles had as great an effect on the wild type allele in the homologue, and in all of these cases the heterochromatin was accompanied by the centromere. MCCIINTOCK's observation relating variegation to the distance from the centromere is based on only five instances, and one of these is of doubtful significance, so that further work on this problem needs to be done.

It was anticipated that the amount of material which has been inserted into the second chromosome to produce $b w^{D}$ might be measured as an increase in the crossover distance in this region. The reduction of crossing over to one third of the normal amount in homozygous $b w^{D}$ was contrary to expectation, and has an important bearing on various theories of crossing over. These observations show that crossing over is not a function of the sum of the tendencies to cross over of each small part of a chromosome. Instead, there is probably a complex interaction between the various parts of the chromosome, each of which has an effect which increases or decreases local crossing over. The very small amount of crossing over which is observed in the heterochromatic regions of Drosophila melanogaster appears to be an expression of a tendency against crossing over. Although this idea is not new, the evidence supporting the idea is. As we do not know that property of the genes or the chromosomes which governs crossing over, and therefore we do not know what the relationship is between mitotic chromosome length and map distance, it might be argued that the apparently large regions of heterochromatin which have so small a crossover length are really short, but are extended in metaphase by the pull of the adjacent centromere. The evidence presented here that the homozygous insertion of this heterochromatin can cause a shortening of the apparent length of adjacent 
euchromatin means that the anti-crossover effect is real. We can more readily accept the chromocentral heterochromatin as being composed of many genes or blocks of genes with this evidence that its short length on the crossover map is due to an anticrossover tendency. There is a slight possibility that $b w^{D}$ influences the whole right arm of chromosome 2 , but the small deficit of observed crossovers would signify that any such influence must be very small to the left of plexus, if it occurs at all. Therefore, the remarkable reduction in crossing over is a local effect, presumably due to action by the homozygous mutant on its immediate neighbors.

The theory of crossing over at or near "frontiers" between euchromatin and heterochromatin, which has been propounded by WHITE (1945) would suggest that the insertion of heterochromatin into a euchromatic region should be accompanied by an increase in crossing over. As this is not so, these observations have a detrimental effect on the value of the frontier theory. However, the theory is sufficiently flexible to preclude the possibility that these findings are fatal to it.

The other crossover data which were studied had held the hope that the position of the brown locus should be localized further than the limits of 59D9 and 59E1.2, which were established by SLATIS (1955). As appropriate markers were present, it would have been possible to place the position of brown within the last three bands of $59 \mathrm{D}$ or the doublet of $59 \mathrm{E}$ with only one crossover. The negative result may be the result of insufficient observation, suppression of crossing over, chromosome structural heterozygosity, too small a distance between bw and the insertion, or the presence of an amorphic allele on the $b w^{D}$ chromosome. The fact that the effect on crossing over which is measured in heterozygotes for $b w^{D}$ is greater to the left of bw than to the right is probably not important in this problem.

\section{SUMMARY}

A reexamination of brown-dominant has resulted in the interpretation of the cytological picture as an insertion of three dark bands and probably one light band between the last band of $59 \mathrm{D}$ and the $59 \mathrm{E}$ doublet. The inserted material looks like chromocentral heterochromatin. Variegation is produced by this insertion, but it can only be seen if other pigment has been removed by genes such as scarlet, and when the fly is heterozygous for a wild allele. These two observations are consistent with other reports on heterochromatic position effects at the brown locus. The interpretation of this mutant as a euchromatic position effect can now be disregarded.

Brown-dominant causes only a slight reduction in crossing over when heterozygous with a wild type chromosome, but crossing over in its immediate vicinity is remarkably reduced in the homozygote. There is little or no effect on the remainder of the chromosome arm. The reduction of crossing over indicates that this inserted heterochromatin has a tendency to block crossing over despite the fact that the amount of chromatin present is greater than normal and the chromosomes are structurally homozygous. Therefore, crossing over cannot be interpreted as the sum of the tendencies to recombine of each of the parts of the chromosome. Instead, it must be assumed that each part of the chromosome contributes some characteristic quality to increase or decrease crossing over and the observed crossovers are the result of an interplay of many factors. 


\section{LITERATURE CITED}

Griffen, A. B., and W. S. Stone, 1940 The $w^{m b}$ and its derivatives. U. Texas Publ. No. 4032 : $190-200$.

Hannah, A., 1951 Localization and function of heterochromatin in Drosophila melanogaster. Advances in Genetics 4: 87-125.

Hinton, T., 1940 Report of Taylor Hinton. Dros. Info. Serv. 13: 49.

1942 Report of T. Hinton. Dros. Info. Serv. 16: 48.

1946 The structure of the bands of salivary gland chromosomes. J. Hered. 37: 98-102.

Hinton, T., and W. Goodsmith, 1950 An analysis of phenotypic reversions at the brown locus in Drosophila. J. Exp. Zool. 114: 103-114.

McClintock, B., 1951 Chromosome organization and genic expression. Cold Spring Harbor Symp. Quant. Biol. 16: 13-47.

Slatis, H. M., 1955 Position effects at the brown locus in Drosophila melanogasler. Genetics 40: $5-23$

White, M. J. D., 1945 Animal Cytology and Evolution. Cambridge: The University Press. 375 pp. 